\title{
Ischemic and non-ischemic patterns of late gadolinium enhancement in heart failure with reduced ejection fraction
}

\author{
Patrycja S. Matusik ${ }^{1}$, Amira Bryll ${ }^{2}$, Paweł T. Matusik ${ }^{3,4}$, Tadeusz J. Popiela ${ }^{2}$ \\ ${ }^{1}$ University Hospital, Department of Diagnostic Imaging, Krakow, Poland \\ ${ }^{2}$ Jagiellonian University Medical College, Faculty of Medicine, Chair of Radiology, Krakow, Poland \\ ${ }^{3}$ The John Paul II Hospital, Department of Electrocardiology, Krakow, Poland \\ ${ }^{4}$ Jagiellonian University Medical College, Faculty of Medicine, Institute of Cardiology, \\ Department of Electrocardiology, Krakow, Poland
}

\begin{abstract}
Background: Late gadolinium enhancement ( $L G E)$ by cardiac magnetic resonance (CMR) may reveal myocardial fibrosis which is associated with adverse clinical outcomes in patients undergoing implantable cardioverter-defibrillator (ICD) placement. At the same time, transmural LGE in the posterolateral wall is related to nonresponse to conventional cardiac resynchronization therapy (CRT). Herein, the aim was to assess the presence and determinants of LGE in CMR in heart failure (HF) with reduced ejection fraction. Methods: Sixty-seven patients were included (17.9\% female, aged 45 [29-60] years), who underwent $L G E-C M R$ and had left ventricular ejection fraction (LVEF) as determined by echocardiography.

Results: In HF patients with LVEF $\leq 35 \%(n=29)$, ischemic and non-ischemic patterns of LGE were observed in $51.7 \%$ and $34.5 \%$ of patients, respectively. In controls $(n=38)$, these patterns were noted in $23.7 \%$ and $42.1 \%$ of patients, respectively. HF patients with $L V E F \leq 35 \%$ and transmural LGE in the posterolateral wall (31.0\%) were characterized by older age, coronary artery disease (CAD) and previous myocardial infarction (MI) $(61 \pm 6$ vs. $49 \pm 16$ years, $p=0.008,100 \%$ vs. $40 \%, p=0.003$ and $78 \%$ vs. $25 \%, p=0.014$, respectively). In patients with $L V E F \leq 35 \%, L G E$ of any type, diagnosed in $86.2 \%$ of patients, was associated with CAD (68\% vs. $0 \%, p=0.02)$, while only trends were observed for its association with older age and previous $M I(p=0.08$ and $p=0.12$, respectively).

Conclusions: Among HF patients with $L V E F \leq 35 \%$, clinical factors including older age, CAD, and previous MI are associated with transmural LGE in the posterolateral wall, while CAD is associated with LGE. This data may have potential implications for planning ICD and CRT placement procedures. (Cardiol J 2021; 28, 1: 67-76)
\end{abstract}

Key words: heart failure, late gadolinium enhancement, ischemic, cardiac magnetic resonance, transmural late gadolinium enhancement, cardiac resynchronization therapy, implantable cardioverter-defibrillator

\section{Introduction}

Appropriate patient evaluation for the placement of implantable cardioverter-defibrillators (ICD) and cardiac resynchronization therapy (CRT) devices is important in the management of heart failure (HF) patients with reduced ejection fraction (HFrEF). In patients with an ischemic or nonischemic etiology of $\mathrm{HF}$, left ventricular ejection fraction (LVEF) $\leq 35 \%$, as determined by echocardiography, continues to be used as a major criterion when considering patients for placement of an ICD

Address for correspondence: Paweł T. Matusik, MD, PhD, FEHRA, Jagiellonian University Medical College, Faculty of Medicine, Institute of Cardiology, Department of Electrocardiology, The John Paul II Hospital, ul. Prądnicka 80, 31-202 Kraków, Poland, tel: +48 1261422 77, fax: +48 1261422 26, e-mail: pawel.matusik@wp.eu 


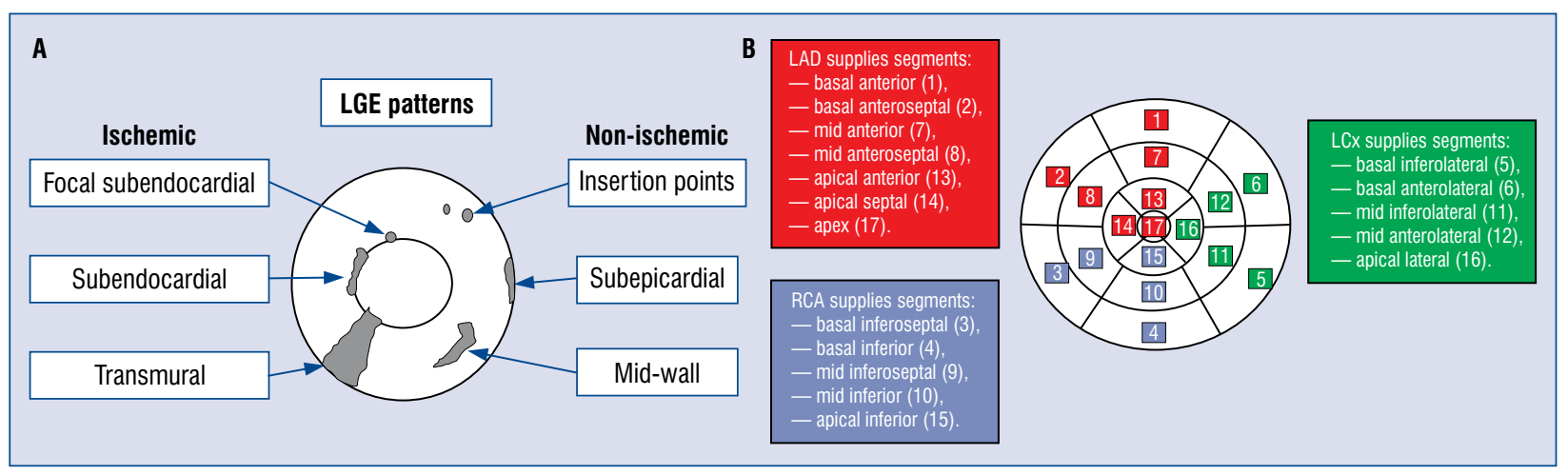

Figure 1. The patterns of late gadolinium enhancement reflecting ischemic and non-ischemic myocardial injury (A) and assignment of the 17 myocardial segments to the territories of the coronary arteries (B). Based on references [7, 11]; LAD — left anterior descending coronary artery; LCx — left circumflex coronary artery; LGE — late gadolinium enhancement; RCA — right coronary artery.

in primary sudden cardiac death (SCD) prevention and to improve HF through CRT [1]. However, effective SCD risk stratification based solely on HF symptoms and LVEF has a number of limitations. At the same time, about $30 \%$ of patients do not respond to CRT $[2,3]$.

Cardiac magnetic resonance (CMR) imaging, especially with late gadolinium enhancement (LGE), is a valuable clinical tool in diagnostic of patients with HF or left ventricular (LV) dysfunction $[4,5]$. Gadolinium-based contrast agents accumulate and demonstrate delayed enhancement within areas of increased extracellular space such as fibrosis, thus enhancing areas of scarring [6]. The patterns of LGE help to differentiate between ischemic and non-ischemic myocardial injury (Fig. 1A). Generally, ischemic myocardial injury tends to cause LGE which is typically subendocardial or transmural [7]. Non-ischemic myocardial injury can be observed at the epicardium, in the midwall, or at insertion points $[5,7,8]$. Moreover, LGE has been found to predict clinical outcomes in selected patients undergoing ICD placement [9]. At the same time, response to conventional CRT is decreased in the presence of transmural scarring in the posterolateral $\mathrm{LV}$ segments (the place over which an LV lead is usually placed) [10].

The cardiac LV segmentation model published by the American Heart Association (AHA) divides the heart into 17 segments and is now widely used for the description of disease-affected myocardium [11]. These 17 segments have a reasonably consistent vascular supply from the main coronary arteries (Fig. 1B). As described previously, generally the left anterior descending coronary artery (LAD) supplies myocardial segments $1,2,7,8,13,14$, and 17 , the right coronary artery (RCA) supplies segments $3,4,9,10$, and 15 , while the left circumflex coronary artery ( $\mathrm{LCx})$ supplies the remainder myocardial segments $(5,6,11,12$, and 16) [11]. However, coronary arteries may be anomalous and their anatomy varies from patient to patient, creating a limitation of the AHA model [12-15].

The main purpose of this study was to assess the presence and determinants of LGE in CMR in patients with $\mathrm{HFrEF}$.

\section{Methods}

\section{Study population}

The study group included 67 patients $(17.9 \%$ female, aged 45 [29-60] years) who underwent LGE-CMR at the Department of Diagnostic Imaging, University Hospital in Krakow between 2011 and 2015, and had data available on LVEF as determined by echocardiography. Further data were obtained from a structured medical documentation review (baseline patient demographics and clinical data including chronic diseases). Diagnosis of significant coronary artery disease (CAD) was based on coronary angiography (available in medical records of 36 patients) and was defined as coronary artery diameter stenosis of $50 \%$ or more, or previous cardiac revascularization (coronary angioplasty or coronary artery bypass grafting) [16]. Moreover, specifically a group of patients with $\mathrm{LVEF} \leq 35 \%$, as determined by echocardiography, was addressed, because they are frequently considered for ICD and CRT placement according 
to the European Society of Cardiology guidelines [17]. The study was approved by the local ethics committee.

\section{CMR imaging}

Cardiac magnetic resonance imaging was performed using a 1.5 Tesla scanner. Steady-state free precession cine images were acquired in the short-axis and two-, three-, and four-chamber views. LGE images were generally acquired $10-20$ min after injection of $0.2 \mathrm{~mL} / \mathrm{kg}$ gadoteridol (ProHance). Inversion recovery time was individually adapted to maximize contrast between regions of LGE and normal myocardium. The cardiac LV segmentation model published by the AHA was used for the description of disease-affected myocardial segments [11]. The distribution of LGE was also assessed according to territories supplied by coronary arteries as proposed in the AHA model [11]. Posterolateral LV segments were defined as basal inferior (4), basal inferolateral (5), mid inferior (10), and mid inferolateral (11) segments [10]. A transmural scar was defined as a hyperenhancement extending $\geq 51 \%$ of $\mathrm{LV}$ wall thickness in $\geq 1$ of the LV segments [10].

\section{Statistical analysis}

Continuous variables are presented as means \pm standard deviations or medians (interquartile ranges $[\mathrm{IQR}]$ ), while categorical variables are shown as numbers and percentages. Associations between categorical variables were assessed using the Pearson chi-squared test or the Fisher's exact test. Continuous variables between two groups were compared using the Student's t-test or Mann-Whitney U test, as appropriate. The Spearman rank test was used to measure the association between two continuous variables (both of the variables tested had a non-normal distribution). Statistical significance was defined as a $\mathrm{p}$ value $<0.05$. Statistical analyses were performed using IBM SPSS Statistics (version 24, IBM Corp., Armonk, NY, USA).

\section{Results}

\section{Study population}

Twenty nine patients with HF and LVEF $\leq 35 \%$, and 38 control patients with LVEF $>35 \%$, as determined by echocardiography, were analyzed. Baseline characteristics of patients are shown in Table 1. In the whole group of patients only ischemic or only non-ischemic patterns of LGE were observed in 24 (35.8\%) and 26 (38.8\%) patients, respectively. Six (9.0\%) patients (only in the control group) had features of both ischemic and non-ischemic or a non-specific LGE pattern, while in $11(16.4 \%)$ patients no LGE was observed. LVEF measured by echocardiography correlated with LVEF measured by CMR $(\mathrm{R}=0.825$, $\mathrm{p}<0.001)$. Median LVEF measured by echocardiography was $47 \%$ (IQR 25-60\%), while median LVEF measured by CMR was 44\% (IQR 25-54\%). Results of coronary angiography were available in medical documentation from the University Hospital for 36 patients. The median time interval between CMR and coronary angiography was 7 days (IQR 3-24 days).

\section{Comparison of HF patients with LVEF} of $35 \%$ or less and controls with LVEF $>35 \%$

Among patients with $\mathrm{HF}$ and LVEF $\leq 35 \%$, as determined by echocardiography, an ischemic pattern of LGE was observed in 15 (51.7\%) subjects, non-ischemic in $10(34.5 \%)$ patients, while $4(13.8 \%)$ patients had no LGE. In controls, an ischemic pattern of LGE was observed in $9(23.7 \%)$ subjects, non-ischemic in $16(42.1 \%)$, combined ischemic and non-ischemic or a non-specific pattern in $6(15.8 \%)$, while $7(18.4 \%)$ had no LGE.

There was no significant difference regarding the presence of any LGE between HF patients with $\mathrm{LVEF} \leq 35 \%$ when compared to controls. However, the ischemic pattern of LGE was more prevalent in $\mathrm{HF}$ patients with $\mathrm{LVEF} \leq 35 \%$ when compared to controls (Table 1). Moreover, in the first group of patients, transmural LGE was observed more frequently when compared with the remainder of the patients $(44.8 \%$ vs. $13.2 \%, \mathrm{p}=0.004)$. The presence of LGE of any pattern was observed more frequently in patients with LVEF $\leq 35 \%$ than in controls in the mid and apical third $(75.9 \%$ vs. $44.7 \%, \mathrm{p}=0.01 ; 51.7 \%$ vs. $26.3 \%, \mathrm{p}=0.033$; respectively), while there was no difference in observed LGE in the basal third between these two groups (Table 2). Interestingly, in the mid third LGE was observed more commonly in segments 8,10 , and 12 , while in the apical third LGE was observed more commonly in segments 13,15 , and 16 in HF patients with LVEF $\leq 35 \%$ than in the control group (Table 2).

In patients for whom coronary angiography data were available $(n=36)$ there was a trend towards a higher prevalence of CAD in HF patients with LVEF $\leq 35 \%$ than the remainder of the patients (63.6\% vs. $35.7 \%, \mathrm{p}=0.102$; Table 3 ). When individual coronary arteries were analyzed, only the LCx was more commonly affected by 
Table 1. Baseline characteristics of heart failure (HF) patients with left ventricular ejection fraction $(\mathrm{LVEF}) \leq 35 \%$ determined by echocardiography and controls with LVEF $>35 \%$.

\begin{tabular}{|c|c|c|c|}
\hline Parameters & $\begin{array}{l}\text { Patients with HF and } \\
\text { LVEF } \leq 35 \%(n=29)\end{array}$ & $\begin{array}{l}\text { Control patients } \\
\qquad(\mathrm{n}=38)\end{array}$ & $\mathbf{P}$ \\
\hline \multicolumn{4}{|l|}{ Demographics } \\
\hline Female sex & $5(17.2 \%)$ & $7(18.4 \%)$ & 0.901 \\
\hline Age [years] & $57.0(38.5-62.0)$ & $35.0(25.8-57.5)$ & 0.004 \\
\hline LVEF determined by echocardiography [\%] & $23.3 \pm 7.3$ & $57.1 \pm 8.8$ & $<0.001$ \\
\hline \multicolumn{4}{|l|}{ Diseases and risk factors } \\
\hline $\mathrm{HF}$ & $29(100.0 \%)$ & $13(34.2 \%)$ & $<0.001$ \\
\hline Myocardial infarction & $12(41.4 \%)$ & $9(23.7 \%)$ & 0.122 \\
\hline CAD & $17(58.6 \%)$ & $15(39.5 \%)$ & 0.120 \\
\hline Atrial fibrillation & $6(20.7 \%)$ & $3(7.9 \%)$ & $0.160^{*}$ \\
\hline Diabetes & $6(20.7 \%)$ & $4(10.5 \%)$ & $0.309 *$ \\
\hline Hyperlipidemia & $13(44.8 \%)$ & $12(31.6 \%)$ & 0.267 \\
\hline Hypertension & $16(55.2 \%)$ & $13(34.2 \%)$ & 0.086 \\
\hline Smoking & $8(27.6 \%)$ & $8(21.1 \%)$ & 0.534 \\
\hline \multicolumn{4}{|l|}{ CMR parameters } \\
\hline CMR-LVEF [\%] & $24.7(19.2-32.4)$ & $51.8(46.6-61.2)$ & $<0.001$ \\
\hline CMR-LVEDV [mL] & $271.3(184.1-368.9)$ & $170.9(140.9-189.7)$ & $<0.001$ \\
\hline CMR-LVESV [mL] & 205.5 (121.5-280.3) & 73.1 (61.7-93.6) & $<0.001$ \\
\hline Ischemic LGE pattern only & $15(51.7 \%)$ & $9(23.7 \%)$ & 0.018 \\
\hline Any LGE & $25(86.2 \%)$ & $31(81.6 \%)$ & $0.745^{*}$ \\
\hline Any LGE in posterolateral LV segment & $14(48.3 \%)$ & $11(28.9 \%)$ & 0.105 \\
\hline Any transmural LGE & $13(44.8 \%)$ & $5(13.2 \%)$ & 0.004 \\
\hline Any transmural LGE in posterolateral segment & $9(31.0 \%)$ & $3(7.9 \%)$ & 0.014 \\
\hline
\end{tabular}

*The Fisher's exact test (exact significance, 2-sided). Values are presented as mean \pm standard deviation or median (interquartile range) or number (percentage). CAD — coronary artery disease; CMR — cardiac magnetic resonance; LGE — late gadolinium enhancement;

LVEDV - left ventricular end diastolic volume; LVESV - left ventricular end systolic volume

significant $\mathrm{CAD}$ in $\mathrm{HF}$ patients with $\mathrm{LVEF} \leq 35 \%$ when compared to controls $(50.0 \%$ vs. $7.1 \%$, $\mathrm{p}=0.011)$. At the same time, when LGE was assessed in segments according to coronary artery distribution, there was a trend to more commonly observed LGE in segments supplied by the LAD in $\mathrm{HF}$ patients with $\mathrm{LVEF} \leq 35 \%$ than in controls $(68.2 \%$ vs. $35.7 \%, \mathrm{p}=0.056$; Table 3$)$.

\section{Assessment of HF patients with LVEF} of $35 \%$ or less with or without LGE

The vast majority of studied HF patients with $\mathrm{LVEF} \leq 35 \%$ had observed LGE $(\mathrm{n}=25,86.2 \%)$. LGE in HF patients with LVEF $\leq 35 \%$ was associated with $\mathrm{CAD}(68.0 \%$ vs. $0.0 \%, \mathrm{p}=0.02)$, while only trends were observed for its association with older age and previous myocardial infarction (MI) (54.4 \pm 13.4 vs. $41.0 \pm 16.2, p=0.08$ and $48.0 \%$ vs. $0.0 \%, \mathrm{p}=0.12$, respectively).

Patients with LVEF $\leq 35 \%$ and transmural LGE were older than the remainder of HF pa- tients with $\mathrm{LVEF} \leq 35 \%$ and no transmural LGE $(60.1 \pm 7.6$ vs. $46.5 \pm 15.7, \mathrm{p}=0.006$; Table 4$)$. $\mathrm{CAD}, \mathrm{MI}$, and the ischemic pattern of LGE were observed more commonly in patients with LVEF $\leq 35 \%$ and transmural LGE when compared with the remainder of patients with $\mathrm{LVEF} \leq 35 \%$ and no transmural LGE (92.3\% vs. $31.3 \%, \mathrm{p}=0.001$; $84.6 \%$ vs. $6.3 \%, \mathrm{p}<0.001 ; 100.0 \%$ vs. $12.5 \%$, $\mathrm{p}<0.001$, as in Table 4).

In $\mathrm{HF}$ patients with $\mathrm{LVEF} \leq 35 \%$, transmural LGE in the posterolateral wall (31\%) was associated with older age, CAD, and previous $\mathrm{MI}(60.6 \pm$ 6.3 vs. $49.0 \pm 15.6$ years, $p=0.008,100 \%$ vs. $40 \%$, $\mathrm{p}=0.003$ and $77.8 \%$ vs. $25 \%, \mathrm{p}=0.014$, respectively, Table 4). In a group of patients with LVEF $\leq 35 \%$ and transmural LGE in the posterolateral LV segments, the presence of an ischemic pattern of LGE was more prevalent when compared with the remaining $\mathrm{HF}$ patients with $\mathrm{LVEF} \leq 35 \%$ and no observed transmural LGE in this region $(100 \%$ vs. $30 \%, \mathrm{p}=0.001$, Table 4 ). 
Table 2. Comparison of localization of late gadolinium enhancement (LGE) between heart failure (HF) patients with left ventricular ejection fraction (LVEF) $\leq 35 \%$ determined by echocardiography and controls with LVEF > 35\%.

\begin{tabular}{|c|c|c|c|}
\hline Segments & $\begin{array}{l}\text { Patients with HF } \\
\text { and LVEF } \leq 35 \% \\
\text { (n = 29) }\end{array}$ & $\begin{array}{l}\text { Control patients } \\
\qquad(\mathrm{n}=38)\end{array}$ & $\mathbf{P}$ \\
\hline Any LGE pattern in basal segments & $18(62.1 \%)$ & $20(52.6 \%)$ & 0.440 \\
\hline Basal anterior (1) & $7(24.1 \%)$ & $5(13.2 \%)$ & 0.246 \\
\hline Basal anteroseptal (2) & $11(37.9 \%)$ & $9(23.7 \%)$ & 0.207 \\
\hline Basal inferoseptal (3) & $13(44.8 \%)$ & $12(31.6 \%)$ & 0.267 \\
\hline Basal inferior (4) & $10(34.5 \%)$ & $7(18.4 \%)$ & 0.134 \\
\hline Basal inferolateral (5) & $9(31.0 \%)$ & $5(13.2 \%)$ & 0.075 \\
\hline Basal anterolateral (6) & $5(17.2 \%)$ & $3(7.9 \%)$ & $0.278 *$ \\
\hline Any LGE pattern in mid segments & $22(75.9 \%)$ & $17(44.7 \%)$ & 0.01 \\
\hline Mid anterior (7) & $9(31.0 \%)$ & $6(15.8 \%)$ & 0.138 \\
\hline Mid anteroseptal (8) & $11(37.9 \%)$ & $6(15.8 \%)$ & 0.039 \\
\hline Mid inferoseptal (9) & $10(34.5 \%)$ & $7(18.4 \%)$ & 0.134 \\
\hline Mid inferior (10) & $11(37.9 \%)$ & $5(13.2 \%)$ & 0.018 \\
\hline Mid inferolateral (11) & $10(34.5 \%)$ & $7(18.4 \%)$ & 0.134 \\
\hline Mid anterolateral (12) & $10(34.5 \%)$ & $3(7.9 \%)$ & 0.006 \\
\hline Any LGE pattern in apical segments & $15(51.7 \%)$ & $10(26.3 \%)$ & 0.033 \\
\hline Apical anterior (13) & $9(31.0 \%)$ & $4(10.5 \%)$ & 0.035 \\
\hline Apical septal (14) & $11(37.9 \%)$ & $7(18.4 \%)$ & 0.074 \\
\hline Apical inferior (15) & $11(37.9 \%)$ & $5(13.2 \%)$ & 0.018 \\
\hline Apical lateral (16) & $8(27.6 \%)$ & $2(5.3 \%)$ & $0.016 *$ \\
\hline Apex (17) & $3(10.3 \%)$ & $1(2.6 \%)$ & $0.308 *$ \\
\hline
\end{tabular}

*The Fisher's exact test (exact significance, 2-sided). Values are presented as number (percentage).

Table 3. Comparison in observed coronary artery disease in coronary angiography and segments with observed late gadolinium enhancement (LGE) according to coronary artery distribution between heart failure (HF) patients with left ventricular ejection fraction (LVEF) $\leq 35 \%$ as determined by echocardiography and controls with LVEF > 35\%.

\begin{tabular}{|c|c|c|c|}
\hline Significant coronary artery disease and LGE & $\begin{array}{l}\text { Patients with HF } \\
\text { and LVEF } \leq 35 \% \\
\text { (n }=22)\end{array}$ & $\begin{array}{l}\text { Control } \\
\text { patients } \\
(n=14)\end{array}$ & $\mathbf{P}$ \\
\hline \multicolumn{4}{|l|}{ Significant coronary artery disease presence } \\
\hline Any coronary artery & $14(63.6 \%)$ & $5(35.7 \%)$ & 0.102 \\
\hline Right coronary artery & $10(45.5 \%)$ & $3(21.4 \%)$ & 0.143 \\
\hline Left circumflex coronary artery & $11(50.0 \%)$ & $1(7.1 \%)$ & $0.011 *$ \\
\hline Left anterior descending coronary artery & $10(45.5 \%)$ & $3(21.4 \%)$ & 0.143 \\
\hline In one coronary artery & $2(9.1 \%)$ & $4(28.6 \%)$ & $0.181 *$ \\
\hline In two coronary arteries & $6(27.3 \%)$ & $0(0.0 \%)$ & $0.062 *$ \\
\hline In three coronary arteries & $6(27.3 \%)$ & $1(7.1 \%)$ & $0.209 *$ \\
\hline \multicolumn{4}{|l|}{ LGE location by coronary arteries territories } \\
\hline LGE location in left anterior descending coronary artery territory & $15(68.2 \%)$ & $5(35.7 \%)$ & 0.056 \\
\hline LGE location in right coronary artery territory & $15(68.2 \%)$ & $8(57.1 \%)$ & 0.501 \\
\hline LGE location in left circumflex coronary artery territory & $12(54.5 \%)$ & $4(28.6 \%)$ & 0.126 \\
\hline LGE location in one coronary artery territory & $5(22.7 \%)$ & $4(28.6 \%)$ & $0.712^{*}$ \\
\hline LGE location in two coronary arteries territories & $5(22.7 \%)$ & $5(35.7 \%)$ & $0.462^{*}$ \\
\hline LGE location in three coronary arteries territories & $9(40.9 \%)$ & $1(7.1 \%)$ & $0.054^{*}$ \\
\hline
\end{tabular}

*The Fisher's exact test (exact significance, 2-sided). Data on coronary angiography were available for 36 patients. Values are presented as number (percentage). 
Table 4. Differences between patients with and without any observed transmural late gadolinium enhancement (LGE) and between patients with and without any observed transmural LGE in posterolateral left ventricular segments, in heart failure (HF) patients with left ventricular ejection fraction $(\mathrm{LVEF}) \leq 35 \%$ determined by echocardiography.

\begin{tabular}{|c|c|c|c|c|c|c|}
\hline Parameters & $\begin{array}{l}\text { Transmural } \\
\text { LGE present } \\
(\mathbf{n}=13)\end{array}$ & $\begin{array}{c}\text { No transmural } \\
\text { LGE } \\
(n=16)\end{array}$ & $\mathbf{P}$ & $\begin{array}{l}\text { Transmural } \\
\text { LGE in post- } \\
\text { erolateral LV } \\
\text { segments } \\
(n=9)\end{array}$ & $\begin{array}{l}\text { No transmural } \\
\text { LGE in post- } \\
\text { erolateral LV } \\
\text { segments } \\
(n=20)\end{array}$ & I \\
\hline \multicolumn{7}{|c|}{ Demographics and other parameters } \\
\hline Female & $3(23.1 \%)$ & $2(12.5 \%)$ & $0.632 *$ & $1(11.1 \%)$ & $4(20.0 \%)$ & $1^{*}$ \\
\hline Age [years] & $60.1 \pm 7.6$ & $46.5 \pm 15.7$ & 0.006 & $60.6 \pm 6.3$ & $49.0 \pm 15.6$ & 0.008 \\
\hline $\begin{array}{l}\text { LVEF determined } \\
\text { by echocardiography [\%] }\end{array}$ & $24.6 \pm 6.9$ & $22.3 \pm 7.6$ & 0.405 & $22.6 \pm 7.2$ & $23.7 \pm 7.4$ & 0.702 \\
\hline $\begin{array}{l}\text { Urgent admission } \\
\text { to hospital }\end{array}$ & $11(84.6 \%)$ & $7(43.8 \%)$ & $0.052^{*}$ & $8(88.9 \%)$ & $10(50.0 \%)$ & $0.096^{*}$ \\
\hline \multicolumn{7}{|l|}{ Diseases and risk factors } \\
\hline CAD & $12(92.3 \%)$ & $5(31.3 \%)$ & 0.001 & $9(100.0 \%)$ & $8(40.0 \%)$ & $0.003 *$ \\
\hline Myocardial infarction & $11(84.6 \%)$ & $1(6.3 \%)$ & $<0.001$ & $7(77.8 \%)$ & $5(25.0 \%)$ & $0.014^{*}$ \\
\hline Diabetes & $2(15.4 \%)$ & $4(25.0 \%)$ & $0.663^{*}$ & $0(0.0 \%)$ & $6(30.0 \%)$ & $0.137^{*}$ \\
\hline Hypertension & $8(61.5 \%)$ & $8(50.0 \%)$ & 0.534 & $5(55.6 \%)$ & $11(55.0 \%)$ & $1^{*}$ \\
\hline Dyslipidemia & $8(61.5 \%)$ & $5(31.3 \%)$ & 0.103 & $5(55.6 \%)$ & $8(40.0 \%)$ & $0.688^{*}$ \\
\hline Smoking & $3(23.1 \%)$ & $5(31.3 \%)$ & $0.697^{*}$ & $2(22.2 \%)$ & $6(30.0 \%)$ & $1^{*}$ \\
\hline Atrial fibrillation & $3(23.1 \%)$ & $3(18.8 \%)$ & $1^{*}$ & $3(33.3 \%)$ & $3(15.0 \%)$ & $0.339 *$ \\
\hline \multicolumn{7}{|l|}{ CMR parameters } \\
\hline CMR-LVEF [\%] & $24.0(18.3-32.4)$ & $24.8(20.9-36.1)$ & 0.809 & $22.8 \pm 7.9$ & $29.0 \pm 10.4$ & 0.120 \\
\hline CMR-LVEDV [mL] & $281.9 \pm 124.8$ & $284.0 \pm 100.7$ & 0.959 & $332.5 \pm 117.7$ & $260.8 \pm 101.6$ & 0.105 \\
\hline CMR-LVESV [mL] & $212.0 \pm 105.8$ & $205.3 \pm 99.7$ & 0.863 & $256.3 \pm 97.7$ & $186.7 \pm 96.6$ & 0.085 \\
\hline Akinesia & $6(46.2 \%)$ & $4(25.0 \%)$ & $0.270 *$ & $4(44.4 \%)$ & $6(30.0 \%)$ & $0.675^{*}$ \\
\hline Dyskinesia & $7(53.8 \%)$ & $8(50.0 \%)$ & 0.837 & $5(55.6 \%)$ & $10(50.0 \%)$ & $1 *$ \\
\hline Hypokinesia & $13(100.0 \%)$ & $14(87.5 \%)$ & $0.488^{*}$ & $9(100.0 \%)$ & $18(90.0 \%)$ & $1 *$ \\
\hline Ischemic LGE pattern only & $13(100.0 \%)$ & $2(12.5 \%)$ & $<0.001$ & $9(100.0 \%)$ & $6(30.0 \%)$ & $<0.001 *$ \\
\hline
\end{tabular}

*The Fisher's exact test (exact significance, 2-sided). Values are presented as number (percentage). For abbreviations - see Table 1

Patients with LVEF $\leq 35 \%$ and observed LGE in any LV segment from the posterolateral wall were older than patients with $\mathrm{LVEF} \leq 35 \%$ and had no observed LGE in this region $(61.5 \pm 9.3 \mathrm{vs}$. $44.3 \pm 13.2, \mathrm{p}<0.001)$. Among patients with LVEF $\leq 35 \%$ and observed LGE in any LV segment from the posterolateral wall, CAD was more frequently present than in the remaining patients with LVEF $\leq 35 \%$ and no observed LGE in this region $(85.7 \%$ vs. $33.3 \%, p=0.004$; Table 5 ). The ischemic pattern of LGE was more common in patients with LGE in the posterolateral LV wall than in the group without LGE in this region $(78.6 \%$ vs. $26.7 \%, \mathrm{p}=$ 0.005). Transmural LGE of any type was observed more commonly in HF patients with LGE in the posterolateral LV wall than in those without LGE in this region $(71.4 \%$ vs. $20.0 \%, p=0.005$, Table 5$)$.
Comparison of patients with ischemic vs. non-ischemic pattern of LGE

When patients with an ischemic $(n=24)$ vs. non-ischemic pattern $(\mathrm{n}=26)$ of LGE were compared, CAD, previous $\mathrm{MI}$, and dyslipidemia were more common in those with the ischemic pattern of LGE (Table 5). Patients with an ischemic pattern of LGE were more commonly admitted urgently to the hospital and had observed akinesia more commonly in CMR $(79.2 \%$ vs. $46.2 \%, p=0.016 ; 41.7 \%$ vs. $15.4 \%, p=0.039$, Table 5 ) when compared to patients with a non-ischemic LGE pattern. Interestingly, in all patients with an ischemic pattern of LGE ( $=24)$, LGE was observed more commonly only in apical segments when compared to patients with a non-ischemic pattern $(70.8 \%$ vs. $30.8 \%, \mathrm{p}=$ $=0.005)$. Moreover, LGE of any pattern in the pos- 
Table 5. Differences between patients with and without observed any late gadolinium enhancement (LGE) in posterolateral left ventricular segments in heart failure (HF) patients with left ventricular ejection fraction (LVEF) $\leq 35 \%$ as determined by echocardiography and comparison of patients with ischemic and non-ischemic pattern of LGE.

\begin{tabular}{|c|c|c|c|c|c|c|}
\hline Parameters & $\begin{array}{l}\text { LGE in post- } \\
\text { erolateral } \\
\text { wall } \\
(n=14)\end{array}$ & $\begin{array}{c}\text { No LGE in post- } \\
\text { erolateral } \\
\text { wall } \\
(n=15)\end{array}$ & 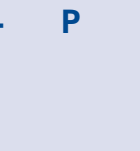 & $\begin{array}{l}\text { Ischemic } \\
\text { LGE pattern } \\
\text { only } \\
(n=24)^{\#}\end{array}$ & $\begin{array}{c}\text { Non-ischemic } \\
\text { LGE pattern } \\
\text { only } \\
(\mathbf{n}=26)^{\#}\end{array}$ & $\mathbf{P}$ \\
\hline \multicolumn{7}{|c|}{ Demographics and other parameters } \\
\hline Female & $1(7.1 \%)$ & $4(26.7 \%)$ & $0.330 *$ & $5(20.8 \%)$ & $4(15.4 \%)$ & $0.721^{*}$ \\
\hline Age [years] & $61.5 \pm 9.3$ & $44.3 \pm 13.2$ & $<0.001$ & $60.0(53.5-68.0)$ & $38.0(27.8-57.5)$ & 0.001 \\
\hline $\begin{array}{l}\text { LVEF determined by } \\
\text { echocardiography [\%] }\end{array}$ & $21.9 \pm 7.5$ & $24.7 \pm 7.0$ & 0.294 & $31.0(20.8-50.0)$ & $50.0(23.8-61.3)$ & 0.147 \\
\hline $\begin{array}{l}\text { Urgent admission } \\
\text { to hospital }\end{array}$ & $10(71.4 \%)$ & $8(53.3 \%)$ & 0.316 & $19(79.2 \%)$ & $12(46.2 \%)$ & 0.016 \\
\hline \multicolumn{7}{|l|}{ Diseases and risk factors } \\
\hline CAD & $12(85.7 \%)$ & $5(33.3 \%)$ & 0.004 & $21(87.5 \%)$ & $9(34.6 \%)$ & $<0.001$ \\
\hline Myocardial infarction & $8(57.1 \%)$ & $4(26.7 \%)$ & 0.096 & $18(75.0 \%)$ & $1(3.8 \%)$ & $<0.001$ \\
\hline Diabetes & $4(28.6 \%)$ & $2(13.3 \%)$ & $0.390^{*}$ & $7(29.2 \%)$ & $3(11.5 \%)$ & $0.164^{*}$ \\
\hline Hypertension & $10(71.4 \%)$ & $6(40.0 \%)$ & 0.089 & $15(62.5 \%)$ & $10(38.5 \%)$ & 0.089 \\
\hline Dyslipidemia & $8(57.1 \%)$ & $5(33.3 \%)$ & 0.198 & $15(62.5 \%)$ & $6(23.1 \%)$ & 0.005 \\
\hline Smoking & $3(21.4 \%)$ & $5(33.3 \%)$ & $0.682^{*}$ & $4(16.7 \%)$ & $8(30.8 \%)$ & 0.243 \\
\hline Atrial fibrillation & $4(28.6 \%)$ & $2(13.3 \%)$ & 0.390 & $5(20.8 \%)$ & $3(11.5 \%)$ & $0.456^{*}$ \\
\hline \multicolumn{7}{|l|}{ CMR parameters } \\
\hline CMR-LVEF [\%] & $24.6 \pm 8.0$ & $29.5 \pm 11.4$ & 0.194 & $31.8 \pm 12.7$ & $45.5 \pm 16.3$ & 0.002 \\
\hline CMR-LVEDV [mL] & $313.4 \pm 113.5$ & $254.8 \pm 102.4$ & 0.155 & $\begin{array}{c}214.9 \\
(169.1-322.4)\end{array}$ & $\begin{array}{c}179.7 \\
(153.3-268.9)\end{array}$ & 0.236 \\
\hline CMR-LVESV [mL] & $229.5 \pm 106.5$ & $188.6 \pm 94.2$ & 0.283 & $\begin{array}{c}140.3 \\
(114.6-260.1)\end{array}$ & $\begin{array}{c}80.8 \\
(61.5-169.0)\end{array}$ & 0.011 \\
\hline Akinesia & $7(50.0 \%)$ & $3(20.0 \%)$ & $0.128 *$ & $10(41.7 \%)$ & $4(15.4 \%)$ & 0.039 \\
\hline Dyskinesia & $8(57.1 \%)$ & $7(46.7 \%)$ & 0.573 & $10(41.7 \%)$ & $7(26.9 \%)$ & 0.272 \\
\hline Hypokinesia & $14(100.0 \%)$ & $13(86.7 \%)$ & $0.483^{*}$ & $21(87.5 \%)$ & $18(69.2 \%)$ & 0.119 \\
\hline Any transmural LGE & $10(71.4 \%)$ & $3(20.0 \%)$ & 0.005 & $17(70.8 \%)$ & $1(3.8 \%)$ & $<0.001$ \\
\hline $\begin{array}{l}\text { Any transmural LGE in } \\
\text { posterolateral LV segment }\end{array}$ & $9(64.3 \%)$ & $0(0.0 \%)$ & $<0.001 *$ & $11(45.8 \%)$ & $1(3.8 \%)$ & $<0.001$ \\
\hline
\end{tabular}

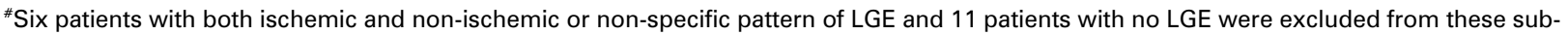
analyses. *Fisher's exact test (exact significance, 2-sided). Values are presented as number (percentage). For abbreviations see Table 1.

terolateral wall was observed more commonly in patients with an ischemic LGE pattern when compared to patients with a non-ischemic LGE pattern $(66.7 \%$ vs. $34.6 \%, \mathrm{p}=0.024)$. Similarly, transmural LGE, including that in the posterolateral wall, was observed more commonly in the first group when compared to the second group (Table 5). Interestingly, only LVEF measured by CMR, but not by echocardiography, differed between patients with an ischemic and non-ischemic pattern of LGE $(31.8 \pm$ \pm 12.7 vs. $45.5 \pm 16.3, \mathrm{p}=0.002 ; 31.0$ [20.8-50.0] vs. 50.0 [23.8-61.3], $\mathrm{p}=0.147$, Table 5).

\section{Discussion}

Cardiac magnetic resonance is currently the most accurate non-invasive method to evaluate myocardial necrosis and fibrosis, which helps in the identification of the underlying cause of $\mathrm{LV}$ dysfunction, and may provide important prognostic information [18-21]. Previous studies have shown the usefulness of CMR imaging qualitative assessment in the diagnostics of CAD [21-23]. It has been suggested that LGE may be useful in excluding significant $\mathrm{CAD}$ in some patients with new-onset 
LV dysfunction in whom there is no data suggestive of ischemic disease [22]. An analysis of LGE distribution is also valuable when differentiating between non-ischemic etiologies of LV dysfunction including dilated cardiomyopathy, cardiac sarcoidosis, myocarditis, hypertrophic cardiomyopathy, cardiac amyloidosis, and Anderson-Fabry disease [6]. However, it should be highlighted that a LGE pattern is not always specific for a particular disease and the inclusion of clinical information is crucial in the diagnostic process.

Patient-specific coronary supply territories may be derived from magnetic resonance angiography, and these territories sometimes differ from those defined by the AHA model of coronary blood supply, which suggests that the 17-segmented model proposed by the AHA may be inaccurate $[12,24]$. A greater prevalence of significant $\mathrm{CAD}$ in the LCx and a trend towards more frequent LGE in segments supplied by the LAD in HF patients with $\mathrm{LVEF} \leq 35 \%$, have been observed. This highlights the potential discrepancies between coronary territories determined by coronary angiography and the presence of LGE on CMR. Moreover, this study suggests that LVEF as measured in CMR, but not in echocardiography, may be associated with patterns of LGE. Previous studies have shown a strong relationship between myocardial fibrosis and worsening of HF [25-27]. In patients with muscular dystrophy, Florian et al. [28] found a correlation between LVEF and LGE extent and a relationship between the LGE pattern and degree of LV systolic dysfunction. However, several studies involving patients with non-ischemic cardiomyopathy showed only a weak or no relationship between the presence of LGE or LGE volume and LV volume and function [29-32].

LGE-CMR imaging could also be useful in the assessment of patients who are bordeline candidates for CRT since absence of LGE is associated with greater CRT response [10]. Previous studies have found a significant correlation between total scar burden and non-response to CRT, and have proposed a dose-response type relationship in these groups, which may predict this outcome [33, 34]. A recent study including patients with dilated cardiomyopathy and CRT found that CRT-defibrillators provided a survival benefit over CRT-pacemakers only in patients with observed LGE [35]. Moreover, implantation of a LV lead over an area with transmural myocardial scarring may result in an ineffective CRT [10]. Thus, clinical determinants of transmural scarring in posterolateral LV segments were also searched for as its identification may lead to a qualification of patients to other forms of physiologic cardiac pacing, including His or paraHis pacing, which would avoid potentially adverse outcomes related to right ventricular pacing [36]. Findings herein, suggest that clinical variables (older age, $\mathrm{CAD}$, and previous $\mathrm{MI}$ ) are associated with transmural posterolateral LGE.

Cardiovascular risk stratification is important and could be improved by the use of biomarkers or data from Holter electrocardiogram recordings [37-39]. Some studies have demonstrated that the presence of LGE predicts poor clinical outcomes such as hospitalization due to HF, fatal ventricular arrhythmias, and SCD, in patients with either an ischemic or non-ischemic etiology of $\mathrm{HF}$ $[8,26,40-44]$. Non-ischemic HF etiology is one of the predictors of LVEF recovery [45]. However, a recently adjusted analysis has shown that major non-ischemic fibrosis was related to worse clinical outcomes than MI [8]. The presence of LGE was found to be associated with appropriate ICD therapy. Among patients with dilated cardiomyopathy and $\mathrm{LVEF} \leq 35 \%$, ICD implantation was associated with a reduction in mortality only among those with LGE [9]. Thus, the present study is important as it explores the determinants of LGE presence. It was found that CAD is associated with LGE in $\mathrm{HF}$ patients with $\mathrm{LVEF} \leq 35 \%$, while previous MI and older age have a tendency to be associated with LGE in these patients. This is in line with observations by other researches who have shown that the presence of LGE was significantly higher in patients with CAD. Moreover, they suggested that CMR is useful for classification of patients with new-onset $\mathrm{HF}$ and LV systolic dysfunction in relation to the presence or absence of CAD [22, 23]. LGE-CMR imaging may provide independent prognostic information beyond LVEF. Thus, the analysis of LGE presence and distribution may improve patient selection and scheduling for ICD implantation in primary prevention of SCD. The association between LGE and ventricular arrhythmias was observed both in studies on patients with mean LVEF $\leq 35 \%$ and in those with mean LVEF $>35 \%$ [43]. Importantly, many SCD occur in patients with LVEF > 35\% [46]. Therefore, it may be hypothesized that some patients with prevalent LGE, risk factors for SCD and LVEF > 35\% could also benefit from primary SCD prevention with ICD placement.

\section{Limitations of the study}

There are some limitations in this study. The current study is a retrospective analysis and in- 
cludes a relatively small group of patients. There is potential over-representation of non-ischemic $\mathrm{HF}$ etiologies in the present cohort due to typical clinical scenarios in which CMR is most commonly used in clinical practice. Information on coronary angiography results was not available for all patients in their medical documentation. Coronary angiography and CMR were not always performed within few days apart. However, the median time interval between these studies was relatively short at 7 days (IQR 3-24 days). Detailed indications for the use of LGE-CMR before potential cardiovascular implantable electronic devices implantation remain to be established in large prospective studies.

\section{Conclusions}

Among HF patients with LVEF of $35 \%$ or less, clinical factors, including older age, CAD, and previous $\mathrm{MI}$ are associated with transmural LGE in the posterolateral wall, while CAD is associated with LGE. This data may have potential implications for planning ICD and CRT placement procedures.

\section{Conflict of interest: None declared}

\section{References}

1. Poole JE. Present guidelines for device implantation: clinical considerations and clinical challenges from pacing, implantable cardiac defibrillator, and cardiac resynchronization therapy. Circulation. 2014; 129(3): 383-394, doi: 10.1161/CIRCULATIONAHA.112.000762, indexed in Pubmed: 24446408.

2. Birnie DH, Tang ASl. The problem of non-response to cardiac resynchronization therapy. Curr Opin Cardiol. 2006; 21(1): 20-26, doi: 10.1097/01.hco.0000198983.93755.99, indexed in Pubmed: 16355025 .

3. Matusik PT. Cardiac resynchronization therapy: potential of left ventricular pacing. Eur Heart J. 2019; 40(21): 1667-1669, doi: 10.1093/eurheartj/ehz348, indexed in Pubmed: 31152550.

4. Kumar A, Bagur R. Cardiac magnetic resonance in clinical cardiology. World J Cardiol. 2015; 7(1): 6-9, doi: 10.4330/wjc.v7.i1.6, indexed in Pubmed: 25632313.

5. Vogel-Claussen J, Rochitte CE, Wu KC, et al. Delayed enhancement MR imaging: utility in myocardial assessment. Radiographics. 2006; 26(3): 795-810, doi: 10.1148/rg.263055047, indexed in Pubmed: 16702455.

6. Tseng WYI, Su MYM, Tseng YHE. Introduction to cardiovascular magnetic resonance: technical principles and clinical applications. Acta Cardiol Sin. 2016; 32(2): 129-144, doi: 10.6515/ /acs20150616a, indexed in Pubmed: 27122944.

7. Thygesen K, Alpert JS, Jaffe AS, et al. Fourth universal definition of myocardial infarction . Eur Heart J. 2019; 40(3): 237-269.

8. Shanbhag SM, Greve AM, Aspelund T, et al. Prevalence and prognosis of ischaemic and non-ischaemic myocardial fibrosis in older adults. Eur Heart J. 2019; 40(6): 529-538, doi: 10.1093/ /eurheartj/ehy713, indexed in Pubmed: 30445559.
9. Gutman SJ, Costello BT, Papapostolou S, et al. Reduction in mortality from implantable cardioverter-defibrillators in nonischaemic cardiomyopathy patients is dependent on the presence of left ventricular scar. Eur Heart J. 2019; 40(6): 542-550, doi: 10.1093/eurheartj/ehy437, indexed in Pubmed: 30107489.

10. Bleeker GB, Kaandorp TAM, Lamb HJ, et al. Effect of posterolateral scar tissue on clinical and echocardiographic improvement after cardiac resynchronization therapy. Circulation. 2006; 113(7): 969-976, doi: 10.1161/CIRCULATIONAHA.105.543678, indexed in Pubmed: 16476852.

11. Cerqueira M, Weissman N, Dilsizian V, et al. Standardized myocardial segmentation and nomenclature for tomographic imaging of the heart. Circulation. 2002; 105(4): 539-542, doi: 10.1161/ hc0402.102975.

12. Zakkaroff C, Biglands JD, Greenwood JP, et al. Patient-specific coronary blood supply territories for quantitative perfusion analysis. Comput Methods Biomech Biomed Eng Imaging Vis. 2018; 6(2): 137-154, doi: 10.1080/21681163.2016.1192003, indexed in Pubmed: 29392098.

13. Tyczyński P, Kukuła K, Pietrasik A, et al. Anomalous origin of culprit coronary arteries in acute coronary syndromes. Cardiol J. 2018; 25(6): 683-690, doi: 10.5603/CJ.a2017.0142, indexed in Pubmed: 29240961.

14. Spałek M, Stępień-Wałek A, Paszkiewicz J, et al. Double left anterior descending artery: Congenital anomaly or normal variant of coronary arteries? Cardiol J. 2017; 24(4): 445-446, doi: 10.5603/CJ.2017.0090, indexed in Pubmed: 28831779.

15. Woźnica A, Tyczyński P, Brzozowski P, et al. Hypertrophic obstructive cardiomyopathy with anomalous left circumflex coronary artery. Kardiol Pol. 2018; 76(7): 1118, doi: 10.5603/ /KP.2018.0141, indexed in Pubmed: 29984817.

16. le Polain de Waroux JB, Pouleur AC, Goffinet C, et al. Combined coronary and late-enhanced multidetector-computed tomography for delineation of the etiology of left ventricular dysfunction: comparison with coronary angiography and contrast-enhanced cardiac magnetic resonance imaging. Eur Heart J. 2008; 29(20): 2544-2551, doi: 10.1093/eurheartj/ehn381, indexed in Pubmed: 18762553.

17. Ponikowski P, Voors AA, Anker SD, et al. 2016 ESC Guidelines for the diagnosis and treatment of acute and chronic heart failure: The Task Force for the diagnosis and treatment of acute and chronic heart failure of the European Society of Cardiology (ESC) Developed with the special contribution of the Heart Failure Association (HFA) of the ESC. Eur Heart J. 2016; 37(27): 2129-2200, doi: 10.1093/eurhearti/ehw128, indexed in Pubmed: 27206819.

18. Mewton N, Liu CY, Croisille P, et al. Assessment of myocardial fibrosis with cardiovascular magnetic resonance. J Am Coll Cardiol. 2011; 57(8): 891-903, doi: 10.1016/j.jacc.2010.11.013, indexed in Pubmed: 21329834.

19. Buckert D, Tibi R, Cieslik M, et al. Myocardial strain characteristics and outcomes after transcatheter aortic valve replacement. Cardiol J. 2018; 25(2): 203-212, doi: 10.5603/cj.a2017.0121.

20. Duncan AM, Francis DP, Gibson DG, et al. Differentiation of ischemic from nonischemic cardiomyopathy during dobutamine stress by left ventricular long-axis function: additional effect of left bundlebranch block. Circulation. 2003; 108(10): 1214-1220, doi: 10.1161/01. CIR.0000087401.19332.B7, indexed in Pubmed: 12939221.

21. McCrohon JA, Moon JCC, Prasad SK, et al. Differentiation of heart failure related to dilated cardiomyopathy and coronary artery disease using gadolinium-enhanced cardiovascular magnetic resonance. Circulation. 2003; 108(1): 54-59, doi: 10.1161/01. CIR.0000078641.19365.4C, indexed in Pubmed: 12821550. 
22. Valle-Muñoz A, Estornell-Erill J, Soriano-Navarro CJ, et al. Late gadolinium enhancement-cardiovascular magnetic resonance identifies coronary artery disease as the aetiology of left ventricular dysfunction in acute new-onset congestive heart failure. Eur J Echocardiogr. 2009; 10(8): 968-974, doi: 10.1093/ejechocard/ jep115, indexed in Pubmed: 19755468.

23. Soriano CJ, Ridocci F, Estornell J, et al. Noninvasive diagnosis of coronary artery disease in patients with heart failure and systolic dysfunction of uncertain etiology, using late gadoliniumenhanced cardiovascular magnetic resonance. J Am Coll Cardiol. 2005; 45(5): 743-748, doi: 10.1016/j.jacc.2004.11.037, indexed in Pubmed: 15734620.

24. Javadi MS, Lautamäki R, Merrill J, et al. Definition of vascular territories on myocardial perfusion images by integration with true coronary anatomy: a hybrid PET/CT analysis. J Nucl Med. 2010; 51(2): 198-203, doi: 10.2967/jnumed.109.067488, indexed in Pubmed: 20080895.

25. Leong DP, Chakrabarty A, Shipp N, et al. Effects of myocardial fibrosis and ventricular dyssynchrony on response to therapy in new-presentation idiopathic dilated cardiomyopathy: insights from cardiovascular magnetic resonance and echocardiography. Eur Heart J. 2012; 33(5): 640-648, doi: 10.1093/eurheartj/ /ehr391, indexed in Pubmed: 22048681.

26. Gulati A, Jabbour A, Ismail TF, et al. Association of fibrosis with mortality and sudden cardiac death in patients with nonischemic dilated cardiomyopathy. JAMA. 2013; 309(9): 896-908, doi: 10.1001/jama.2013.1363, indexed in Pubmed: 23462786.

27. Wong TC, Piehler KM, Zareba KM, et al. Myocardial damage detected by late gadolinium enhancement cardiovascular magnetic resonance is associated with subsequent hospitalization for heart failure. J Am Heart Assoc. 2013; 2(6): e000416, doi: 10.1161/ /JAHA.113.000416, indexed in Pubmed: 24249712.

28. Florian A, Ludwig A, Engelen M, et al. Left ventricular systolic function and the pattern of late-gadolinium-enhancement independently and additively predict adverse cardiac events in muscular dystrophy patients. J Cardiovasc Magn Reson. 2014; 16: 81, doi: 10.1186/s12968-014-0081-1, indexed in Pubmed: 25315351.

29. Matoh F, Satoh H, Shiraki K, et al. Usefulness of delayed enhancement magnetic resonance imaging to differentiate dilated phase of hypertrophic cardiomyopathy and dilated cardiomyopathy. J Card Fail. 2007; 13(5): 372-379, doi: 10.1016/j.cardfail.2007.02.001, indexed in Pubmed: 17602984.

30. Bohl S, Wassmuth R, Abdel-Aty H, et al. Delayed enhancement cardiac magnetic resonance imaging reveals typical patterns of myocardial injury in patients with various forms of non-ischemic heart disease. Int J Cardiovasc Imaging. 2008; 24(6): 597-607, doi: 10.1007/s10554-008-9300-x, indexed in Pubmed: 18344061.

31. Satoh H, Sano M, Suwa K, et al. Distribution of late gadolinium enhancement in various types of cardiomyopathies: Significance in differential diagnosis, clinical features and prognosis. World J Cardiol. 2014; 6(7): 585-601, doi: 10.4330/wjc.v6.i7.585, indexed in Pubmed: 25068019.

32. Choi DS, Ha JW, Choi B, et al. Extent of late gadolinium enhancement in cardiovascular magnetic resonance and its relation with left ventricular diastolic function in patients with hypertrophic cardiomyopathy. Circ J. 2008; 72(9): 1449-1453, doi: 10.1253/ /circj.cj-07-0874, indexed in Pubmed: 18724020.

33. Ypenburg C, Roes SD, Bleeker GB, et al. Effect of total scar burden on contrast-enhanced magnetic resonance imaging on response to cardiac resynchronization therapy. Am J Cardiol. 2007;
99(5): 657-660, doi: 10.1016/j.amjcard.2006.09.115, indexed in Pubmed: 17317367.

34. Adam RD, Shambrook J, Flett AS. The prognostic role of tissue characterisation using cardiovascular magnetic resonance in heart failure. Card Fail Rev. 2017; 3(2): 86-96, doi: 10.15420/ cfr.2017:19:1, indexed in Pubmed: 29387459.

35. Leyva F, Zegard A, Acquaye E, et al. Outcomes of cardiac resynchronization therapy with or without defibrillation in patients with nonischemic cardiomyopathy. J Am Coll Cardiol. 2017; 70(10): 1216-1227, doi: 10.1016/j.jacc.2017.07.712, indexed in Pubmed: 28859784.

36. Matusik PT. Adverse clinical outcomes related to right ventricular pacing. Eur Heart J. 2019; 40(20): 1586-1588, doi: 10.1093/ eurheartj/ehz279, indexed in Pubmed: 31111886.

37. Matusik PT. Biomarkers and cardiovascular risk stratification. Eur Heart J. 2019; 40(19): 1483-1485, doi: 10.1093/eurheartj/ ehz265, indexed in Pubmed: 31087049.

38. Matusik PS, Matusik PT, Stein PK. Heart rate variability in patients with systemic lupus erythematosus: a systematic review and methodological considerations. Lupus. 2018; 27(8): 1225-1239, doi: 10.1177/0961203318771502, indexed in Pubmed: 29697012.

39. Matusik PT, Prior SM, Butenas S, et al. Association of cardiac troponin I with prothrombotic alterations in atrial fibrillation. Kardiol Pol. 2018; 76(7): 1106-1109, doi: 10.5603/KP.2018.0134, indexed in Pubmed: 29984810.

40. Assomull RG, Prasad SK, Lyne J, et al. Cardiovascular magnetic resonance, fibrosis, and prognosis in dilated cardiomyopathy. J Am Coll Cardiol. 2006; 48(10): 1977-1985, doi: 10.1016/j. jacc.2006.07.049, indexed in Pubmed: 17112987.

41. Kwon DH, Obuchowski NA, Marwick TH, et al. Jeopardized myocardium defined by late gadolinium enhancement magnetic resonance imaging predicts survival in patients with ischemic cardiomyopathy: impact of revascularization. J Am Heart Assoc. 2018; 7(22): e009394, doi: 10.1161/JAHA.118.009394, indexed in Pubmed: 30571486.

42. Di Bella G, Siciliano V, Aquaro GD, et al. Scar extent, left ventricular end-diastolic volume, and wall motion abnormalities identify high-risk patients with previous myocardial infarction: a multiparametric approach for prognostic stratification. Eur Heart J. 2013; 34(2): 104-111, doi: 10.1093/eurheartj/ ehs037, indexed in Pubmed: 22368185.

43. Di Marco A, Anguera I, Schmitt M, et al. Late gadolinium enhancement and the Risk for ventricular arrhythmias or sudden death in dilated cardiomyopathy: systematic review and meta-analysis. JACC Heart Fail. 2017; 5(1): 28-38, doi: 10.1016/j. jchf.2016.09.017, indexed in Pubmed: 28017348.

44. Brown PF, Miller C, Di Marco A, et al. Towards cardiac MRI based risk stratification in idiopathic dilated cardiomyopathy. Heart. 2019; 105(4): 270-275, doi: 10.1136/heartjnl-2018-313767, indexed in Pubmed: 30377260.

45. Agra Bermejo R, Gonzalez Babarro E, López Canoa JN, et al. Heart failure with recovered ejection fraction: Clinical characteristics, determinants and prognosis. CARDIOCHUS-CHOP registry. Cardiol J. 2018; 25(3): 353-362, doi: 10.5603/CJ.a2017.0103, indexed in Pubmed: 28980289.

46. Goldberger JJ, Buxton AE, Cain M, et al. Risk stratification for arrhythmic sudden cardiac death: identifying the roadblocks. Circulation. 2011; 123(21): 2423-2430, doi: 10.1161/CIRCULATIONAHA.110.959734, indexed in Pubmed: 21632516. 